\title{
Where to locate wavelength conversion in WDM networks?
}

\author{
M.Garnot, P. A. Perrier \\ Alcatel Alsthom Corporate Research Center \\ c/o Alcatel Alsthom Recherche \\ Route de Nozay - 91460 Marcoussis (France) \\ tel : (33) 169631360 \\ fax : (33) 169631865 \\ e-mail : Mathieu.Gamot@aar.alcatel-alsthom.fr
}

\begin{abstract}
The massive deployment of Wavelength-Division Multiplexing (WDM) in existing transport networks worldwide will facilitate the definition of a photonic transport layer. Different routing strategies of optical paths in such networks have been investigated [1] ; resource allocation has also been proposed using two different schemes based on wavelength routing and wavelength switching, respectively. The design of a WDM transport network not only implies identifying algorithms for routing and resource allocation but also modeling of different routing functions at the node and network levels. Such a function is wavelength conversion.
\end{abstract}

This paper addresses the use of wavelength conversion in WDM networks.

\section{Introduction : A transport network overview}

The introduction of WDM [2,3] in telecommunication networks is today a worldwide concern. WDM is perceived as a simple, modular and cost-effective technique to enhance the capacity of existing networks through a more efficient use of the available bandwidth of optical fibers.

In addition to the mere capacity increase it offers, the extensive deployment of WDM point-to-point transmission systems opens up the possibility to efficiently perform networking functions directly in the optical domain. With each of the transported signals assigned a distinct wavelength (wavelength channel), carried from node to node on the same fiber, it is possible using space-division and wavelength-division switching techniques to route each channel individually without any synchronization or opto-electronic conversion. New optical routing elements, such as Optical AddDrop Multiplexers (OADMs) and Optical Crossconnects (OXCs), can thus be designed to only extract channels that have reached their destination, and let the others, possibly multiplexed with locally added channels, continue transparently to the following nodes. Many experimental implementations of OXCs and OADMs have already been demosntrated [4] which can be classified into two major classes: Wavelength Switching or Translating (WT) and Wavelength Routing (WR), depending whether wavelength converters are used or not.

\section{General benefits of wavelength conversion}

The original version of this chapter was revised: The copyright line was incorrect. This has been corrected. The Erratum to this chapter is available at DOI: 10.1007/978-0-387-35398-2_19 


\section{Part Two: Wavelength conversion and assignment}

The difference between WR and WT nodes is depicted in Figure 1. In a WR network an optical path is assigned a single wavelength from source to destination. Given that the set of available wavelengths is, in practice, limited (e.g., 32), these wavelengths will have to be reused throughout the network to establish all the required connections provided that two optical paths at the same wavelength do not share a common fiber. In this scheme, wavelength contention can be alleviated only by allocating the optical paths with a global view of all the connections.

In a WT network, wavelength is considered a physical transport resource with a local significance which can be reused from link to link between source and destination. An optical path is therefore assigned - not a single wavelength,- but a concatenation of several, possibly different, wavelengths. Here, wavelength contention can be solved simply by performing wavelength at the nodes.

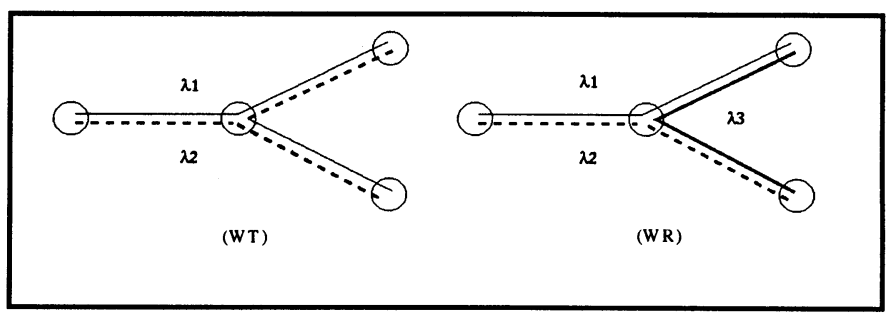

Figure 1:Wavelength Routing (WR) vs

Wavelength Translating (WT) networking schemes

The extent to which wavelength translation is required has given rise to heated debates. Intuitively, however, wavelength conversion can save resources (fewer fibers and smaller nodes) and simplify the management of optical networks [1,5]. The gain to be expected will depend on the network's characteristics (topology, number of nodes, diameter, etc) and the supported traffic (of the optical layer). The results, reported below, on a Pan-American Optical Network [6] show the benefits of wavelength translation [1] in the case of a real network. Though general analytical expressions have been given for regular topologies [7], such expressions for existing networks remain to be determined. Nevertheless, some trends can be derived and explained. For example, it has been found that wavelength conversion is more useful in large-scale or low-connectivity networks when the number of wavelengths is large or when traffic is changing dynamically.

From a physical point of view, it was shown that some all-optical wavelength converters regenerate the Optical Signal-Noise-Ratio. This specific feature can be very attractive in order to optimize the WDM network design. However, the number of all-optical wavelength converters that can be cascaded will be limited by jitter accumulation introduced during the conversion process. This actual drawback concerns only the all-optical implementation of the wavelength conversion ; other implementations (i.e., optoelectronic) can be envisioned that do not suffer from this drawback.

\section{Modeling of wavelength conversion}

According to the traffic, the network topology, and the protection schemes [8], the dimensioning process establishes the end-to-end optical paths and the associated resources. The (optical) traffic matrix is derived from the transport traffic matrix by grooming and multiplexing. The optical network 
topology comprises all the optical network elements which are involved in the transport and routing functions of the WDM layer. We define this topology as a graph in which the vertices represent the optical routing nodes and the axes the fiber links.

The problem is then to assign the appropriate resources to the given paths. It can be shown that resource assignment is an NP-complete (Non-deterministic Polynomial $[9,10]$ ) problem when wavelength converters are used everywhere in the network. Actually, the severe constraint of wavelength continuity (WR) when wavelength conversion is not used turns the wavelength assignment problem into a graph colorability problem [11], which is NP-complete. The problem is not NPComplete only when wavelength conversion is used at every node in the network. Therefore, introducing wavelength conversion only in a few routing nodes does not change the nature of the allocation problem. As a matter of fact, and paradoxically, the addition of wavelength conversion can result in an increase of the number of required resources (fiber, node size, etc).

Unfortunately, for cost reason and optimal benefits, the use of wavelength conversion will be probably restricted to a few routing nodes. Also, we apply the classical wavelength allocation algorithm [11] in order to assign wavelengths to sections of the optical paths (sub-paths). A sub-path is defined as the part of an optical path which does not cross wavelength translating nodes. The following figure shows an end-to-end optical path defined by the traffic matrix and the different sub-paths determined by the addition of wavelength conversion.

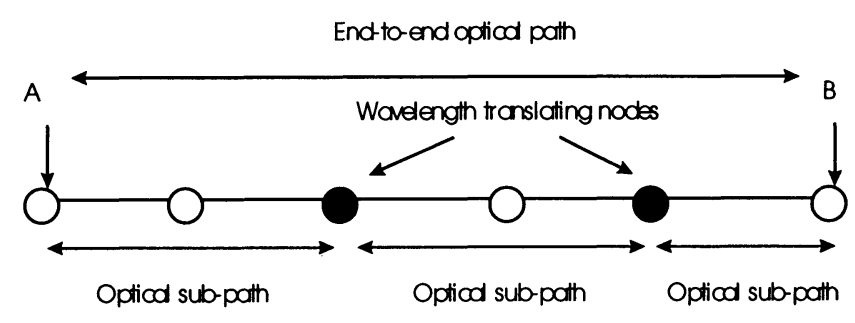

Figure $2:$ Paths and sub-paths

In the wavelength allocation algorithm, each sub-path is treated individually; the wavelength assigned to that sub-path can be different from the wavelengths assigned to the other sub-paths even though all sub-paths belong to the same end-to-end path.

\section{How to select the nodes with wavelength conversion}

The locations at which wavelength conversion will take place is a key point to investigate. Actually, the criteria to select these locations are based on wavelength blocking and on the number and length of the generated sub-paths.

Wavelength blocking can be estimated by analyzing different traffic configurations. This analysis leads to different mathematical models which are not always practical to rapidly obtain dimensioning results. Therefore, we have defined simple criteria to choose the best suited location of wavelength converting 


\section{Part Two: Wavelength conversion and assignment}

nodes, resulting in a better understanding of the global behavior of the WDM optical layer.. These criteria, applied to each routing nodes, are :

- criterion $\mathrm{A}$ : number of optical channels in transit

- criterion B : (number of optical channels in transit)*(node connectivity)

- criterion $\mathrm{C}$ : output fiber utilization ratio

The number but more importantly the length, determined by the number of used links, of the generated sub-paths imposes the introduction of wavelength conversion in the longer (sub-) paths. It follows that criterion A can be modified into criterion $A^{\prime}$ : number of optical channels in transit corresponding to an optical (sub-) path longer than a minimum from end-to-end.

\section{Application and results}

The test network (Figure 3) is the Pan-American Network [6] which interconnects the main cities (maximum number of nodes $=78$ ) in the United-States. This example is interesting since it proposes a large scale network with different traffic configurations. Actually, the traffic is mainly transversal (from East to West and West to East), with high density around the large metropolis (Chicago, NewYork, Dallas, ...).

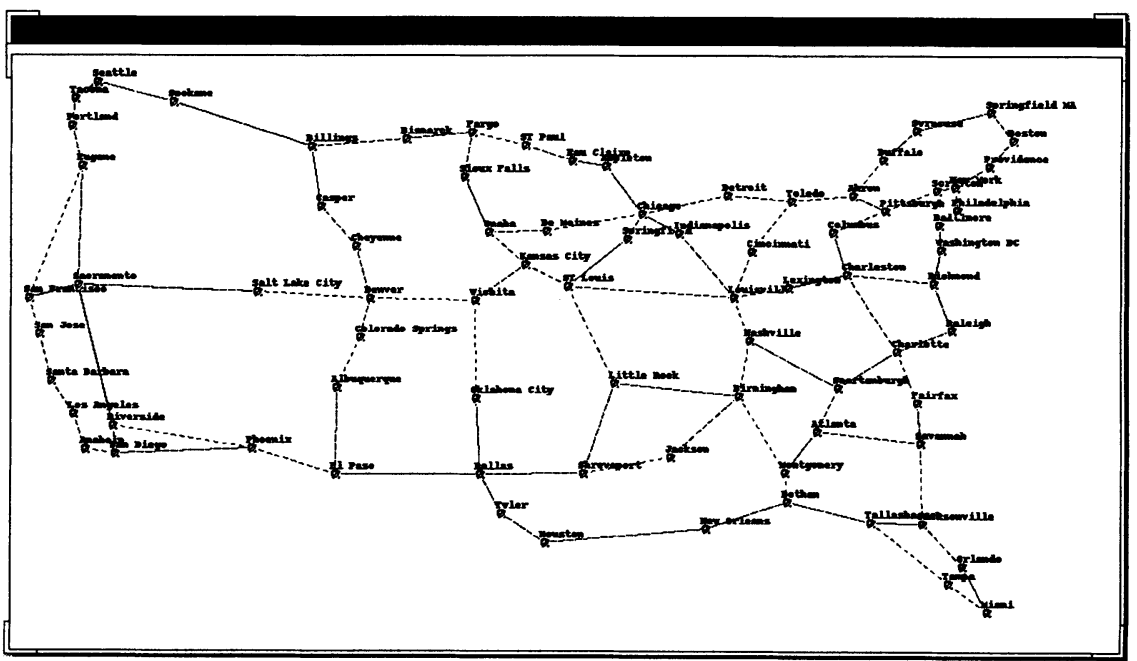

Figure 3 : Pan-American network

Using optical cross-connects without wavelength conversion, the dimensioning results lead to about 88 000 miles of fiber (maximum of 8 wavelengths per fiber; maximum bit rate of $2.5 \mathrm{Gbit} / \mathrm{s}$ on each wavelength) for a traffic matrix containing 466 connections.

The results when the different criteria are applied are presented in figure 4, where the total fiber length is given as a function of the number nodes fully equipped with wavelength converters. 


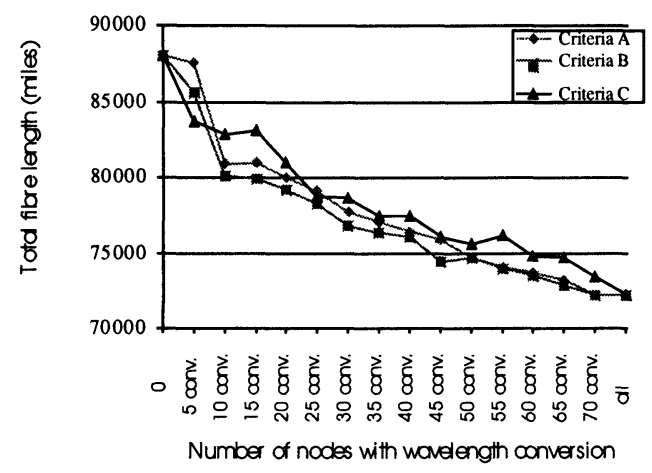

Figure 4 : Total fiber length as a function of the number nodes with wavelength conversion

The figure shows first that the application of the different criteria leads to a significant reduction (about $15 \%$ ) in the total fiber length required, and second that some criteria are more efficient (less number of nodes with wavelength conversion for a given reduction of the fiber length). For example by introducing 15 nodes with wavelength conversion, there is a difference of about 3000 miles ( 3 to $4 \%$ ) between criteria $\mathrm{B}$ and $\mathrm{C}$.

It also shows the possible increase of the required fiber length when more nodes with wavelength conversion are introduced (from 10 to 15 nodes with criterion C). This behavior illustrates the NPcompleteness of the wavelength allocation problem when wavelength conversion is not used everywhere in the network.

\section{Conclusion}

This paper briefly reviews the interest of the WDM technology introduction in transport networks. Our study focuses on the use of wavelength conversion, one of the most attractive feature of the future WDM transport layer. Different criteria have been proposed to select the best locations to introduce this function. It was noticed that the wavelength allocation problem is always NP-complete if only a few routing nodes use wavelength conversion. For this reason, the criteria are based on wavelength blocking and wavelength allocation algorithm with the taget to minimize the number of long sub-paths. We found that the network connectivity is one of the most important parameter (criterion B), but no theoretical formalism can be proposed to this date.

\section{References}

[1] M. Garnot et al., "Routing strategies for optical paths in the WDM networks", ICC'97, Montreal, June 8-12,, pp. 422-426.

[2] C. A. Brackett, "Dense wavelength division multiplexing networks: principles and applications", IEEE J.on Sel. Areas in Comm., vol. 8, no. 6, pp. 948-964, Aug. 1990.

[3] P. Demeester et al., "Photonic transport networks: why, how and when ?", NOC'96, vol. 1, Heidelberger, June, pp. 158-165.

[4] A. Jourdan et al., " Design and implementation of a fully reconfigurable all-optical crossconnect for high capacity multiwavelength transport network", IEEE J. of Lightwave Technology, vol. 14, no. 6, pp. 1198-1206, June 1996.

[5] N. Wauters et al., "Reduction of wavelength blocking through partitioning with wavelength converters", OFC'97, Dallas, Feb., pp. 122-123.

[6] M.Garnot et al., " Dimensioning and design of the WDM optical layer in transport network" , SPIE conference on All-Optical Communication : Architecture, Control, and Network Issues III, November 2-3, 1997, pp. 244-252. 
36 Part Two: Wavelength conversion and assignment

[7] R. A. Barry, P. Humblet, "Models of blocking probability in all optical networks with and without wavelength changers", IEEE INFOCOM'95, Boston,.

[8] L. Nederlof et al., "A comparison of survivability methods for the optical layer", NOC'97, June 17-20, Antwerp, Belgium, pp. 103-110.

[9] M. R. Garey and D. S. Johnson, Computers and intractablility: a guide to the theory of the NP-Completeness, Freeman Publishers, 1979.

[10] B.M.E. Moret and H.D. Shapiro, Algorithms from P to NP, The Benjamin / Cummings Publishing Company, 1991.

[11] I. Chlamtac et al., "Lighpath Communication: An Approach to High Bandwidth Optical WAN's", IEEE Transactions on Communications, vol. 40, no. 7, July 1992, pp. 1171-1182. 